\title{
How Depressive Symptoms Hinder Positive Information Processing: An Experimental Study on the Interplay of Cognitive Immunisation and Negative Mood in the Context of Expectation Adjustment
}

\author{
Tobias Kube $^{1}$ (D) Julia Anna Glombiewski ${ }^{1}$ \\ Accepted: 4 December 2020 / Published online: 22 December 2020 \\ (c) The Author(s) 2020
}

\begin{abstract}
Background People with depression maintain negative expectations despite disconfirming positive experiences by reappraising or discarding novel positive information, referred to as "cognitive immunisation". A second body of literature suggests that negative mood can negatively affect information processing. Bridging these two lines of research, the present study examined the interplay of cognitive immunisation and negative mood in the context of expectation modification.

Methods In a student sample $(N=152)$, we used a well-established experimental paradigm to examine the adjustment of performance expectations in response to positive performance feedback, and its relation to depressive symptoms. In a $2 \times 2$ design, participants received either a negative mood induction, a cognitive immunisation manipulation, both, or no further manipulation.

Results Participants from all experimental groups revised their previous expectations significantly in line with positive performance feedback. However, depressive symptoms were a negative predictor of expectation adjustment, and a moderation analysis indicated that this effect was particularly pronounced if participants underwent the negative mood induction. Conclusions Consistent with previous work, depressive symptoms were associated with a reduced ability to integrate positive information. Furthermore, our results suggest that the activation of negative mood in people with elevated levels of depression may hamper learning from new positive experience.
\end{abstract}

Keywords Depression $\cdot$ Expectation $\cdot$ Cognitive immunisation $\cdot$ Mood $\cdot$ Optimistic bias

\section{Introduction}

Based on well-established findings into the tremendous impact of expectations on perception, emotion, and wellbeing, a relatively nascent area of research in the cognitive sciences has begun to investigate how people adjust their expectations in light of disconfirmatory evidence (Sharot \& Garrett, 2016). For instance, researchers have begun to relate anomalies in belief updating to psychopathological

Supplementary Information The online version contains supplementary material available at https://doi.org/10.1007/s1060 8-020-10191-4.

Tobias Kube

kube@uni-landau.de

Pain and Psychotherapy Research Lab, University of Koblenz-Landau, Ostbahnstr. 10, 76829 Landau, Germany dysfunctions, which has been of particular interest in research on depression. Several lines of research have provided evidence for the hypothesis that depression is related to difficulty in revising negative expectations after receiving unexpectedly positive information, such as: research on interpretation biases (Everaert et al., 2018; Liknaitzky, Smillie, \& Allen, 2017); cognitive inflexibility (Stange et al., 2017); (lack of) optimism bias (Garrett et al., 2014; Korn et al. 2014); and reward insensitivity (Eshel \& Roiser, 2010).

Recently, another mechanism explaining the persistence of negative expectations despite positive experiences in depression has been introduced. According to the concept of "cognitive immunisation" against disconfirmatory evidence (Rief et al., 2015), people with depression tend to reinterpret unexpectedly positive information in such a way that 
the positive information is devalued. ${ }^{1}$ Of note, this concept has similarities with consistency theories from cognitive and social psychology, of which Festinger's (1962) theory of cognitive dissonance is among the most important. According to this theory, holding conflicting beliefs is perceived as being aversive, resulting in the preference to reduce this cognitive dissonance by cognitive or behavioural strategies. For example, in a depressed person holding the core belief "I am incapable", new information suggesting that one did well would be assumed to create dissonance as new information is inconsistent with the core belief, resulting in the desire to reduce cognitive dissonance. This is where the idea of cognitive immunisation comes into play: According to Rief et al. (2015), cognitive immunisation refers to the very process of reappraising new information in such a way that the discrepancy between the prior belief and new information is reduced. It has been suggested that this cognitive reappraisal leads to sustained negative expectations that become immune to belief updating and learning from new experience (Kube et al., 2020; Rief \& Joormann, 2019). In a series of experimental studies, we confirmed this hypothesis by first demonstrating that people with depression maintained negative expectations for their performance despite positive performance feedback, whereas healthy people adjusted their expectation in accordance with the positive feedback (Kube, Rief, Gollwitzer, Gärtner, et al., 2019a, b, c; Kube, Rief, Gollwitzer, et al., 2018). Subsequently, we found that promoting the engagement in cognitive immunisation prevented people with depression from utilising positive information to alter their expectations, while inhibiting the use of cognitive immunisation strategies facilitated the adjustment of negative expectations (Kube, Glombiewski, et al., 2019a, b, c; Kube, Rief, et al., 2019a, b, c). Yet, while there are first indications for the importance of cognitive immunisation in the context of expectation modification, it is not clear whether this cognitive process is specific to depression, or whether it can also be triggered in a non-clinical sample. Therefore, this study aimed to examine whether this style of negatively reappraising unexpected positive information leading to sustained negative expectations can be mimicked in a non-clinical sample through an immunisation-promoting experimental manipulation. In other words, we aimed to investigate if the ability of non-depressed individuals to modify their expectations in accordance with positive feedback would be reduced when a negative reappraisal of positive feedback was promoted. Findings from this study would also add to the literature on cognitive biases in non-clinical samples. For example, early studies in non-clinical samples

\footnotetext{
${ }^{1}$ Notably, cognitive immunisation can refer to both reappraisal of positive and negative information; in this article, we use the term only in the context of devaluing positive information.
}

have found that negative biases (i.e., biases in relation to attention, interpretation, and memory) could be induced in healthy volunteers (Mathews \& Mackintosh, 2000), whereas more recent work has provided mixed findings in this regard (for reviews, see Hertel and Mathews (2011) and Hallion and Ruscio (2011)).

A secondary interest was the role of negative mood in the context of expectation modification. From the general psychology literature, it is known that the presence of negative affect ${ }^{2}$ can hinder information processing. Specifically, it has been shown that negative mood impairs learning and transfer effects (Brand et al., 2007) as well as positive feedback processing (Hammer \& Stone-Romero, 1996). Further evidence arises from Ziegler's mood-congruent expectancies approach, speaking to impaired information processing if mood-incongruent information is received (Ziegler, 2010, 2013). Thus, we hypothesised that the induction of negative mood hinders the revision of negative performance expectations after positive performance feedback, although we are cognizant of other studies that did not find adverse effects of negative mood on information processing, as summarised by Forgas (2013). In accordance with the aforementioned negative effects of negative affect on information processing, other research has found bidirectional effects of negative mood and the engagement in maladaptive cognitive response styles (i.e. rumination) in both clinical and non-clinical samples (Kuehner et al., 2009; Lyubomirsky et al., 1998; NolenHoeksema et al., 1993; Nolen-Hoeksema et al., 1994; Yoon \& Joormann, 2012). That is, the activation of negative mood can trigger the occurrence of negative thinking, and conversely, maladaptive rumination can amplify negative mood. In view of this bidirectional relationship between negative mood and rumination, we investigated how the combination of a negative mood induction and a cognitive immunisation manipulation affects belief updating. More specifically, we hypothesised that if participants are already in a negative mood, a manipulation promoting negative appraisal might further contribute to a negative affective-cognitive state in which the integration of novel positive information into previous beliefs is particularly hampered. Moreover, with reference to research relating depressive mood to the engagement in dysfunctional cognitive processes (Nolen-Hoeksema et al., 1993), we predicted that participants with elevated depressive symptoms would show little adjustment of negative expectations if they undergo a negative mood induction before receiving unexpectedly positive feedback. If this hypothesis were confirmed, it may have implications for the conduction of interventions relying on learning from new experiences (such as behavioural experiments), since the

\footnotetext{
2 The terms "mood" and "affect" are often used in an interchangeable way. Here, we use them interchangeably too.
} 
success of such an intervention could be impaired if patients are in negative mood while learning.

We tested these hypotheses using a $2 \times 2$ design, which allowed us to examine both main and interaction effects of mood and cognitive immunisation. In particular, there were four groups: induction of negative affect (Affect); promotion of cognitive immunisation (Immunisation); combination of mood induction and cognitive immunisation manipulation (Immunisation + Affect); and a control group receiving neither an affect nor an immunisation manipulation. With regard to the role of depressive symptoms, we expected that depressive symptoms would predict reduced expectation adjustment, and additionally tested the moderation hypothesis that the negative effect of depressive symptoms on the modification of previous expectations would be particularly pronounced in the two groups that underwent the negative mood induction.

\section{Methods}

The study was approved by the local ethics committee (reference number 151_2018) and was conducted in accordance with ethical standards as laid down in the 1964 Declaration of Helsinki and its later amendments. All participants gave written informed consent.

\section{Participants}

An a-priori power analysis using $\mathrm{G} *$ Power, expecting small to medium effects of the experimental manipulations (expected $f=0.14$; alpha $=0.05$; power $=0.05$; four groups; two measurements; correlation between the two measurements: $r=0.50$ ) indicated a minimum sample size of 144 participants. A total of 157 people participated in the study. Participants were students from the University of KoblenzLandau, who were recruited via university email lists. Inclusion criteria were: at least 18 years old and sufficient German language skills. Participants received course credit for their participation or, alternatively, $9 €$ as financial compensation.

\section{Procedure}

\section{Cover Story}

In order to conceal the actual aim of the study and to avoid demand effects, participants were first informed that the aim of the study was to investigate the relationship between mood and performance. As part of the cover story, participants were told that half of the participants would watch a brief film sequence which normally induces feelings of sadness. Subsequently, participants were informed that they would have to take a performance test which they would not know yet and was very difficult. The last part of this cover story has already been used in several previous studies (Kube, Glombiewski, et al., 2019a, b, c; Kube, Rief, et al., 2019a, b, c; Kube et al., 2018) with the aim of lowering participants' initial expectations for their performance.

\section{Randomisation and Blinding}

Prior to each experimental session, participants were randomly assigned to one of the four groups. Participants were only insofar aware of their assignment as they knew that half of the participants would have to watch a brief film sequence, while the other half would not. They did not know that the study was investigating not only mood but also cognitive immunisation, and that there was a total of four groups. The experimenters were aware of participants' group assignment.

\section{Mood Induction vs. Distraction}

After the first assessment of expectations, all participants completed a first rating of their current mood. Participants who were randomised to the conditions Affect or Immunisation + Affect next watched a brief sequence taken from the film "The Champ" from 1979. The sequence lasting $2 \mathrm{~min}$ and $45 \mathrm{~s}$ shows a professional boxer dying after a fight in front of his little crying son. It has been frequently used in emotion research and has been shown to be suitable for inducing negative mood (Gross \& Levenson, 1995; Rottenberg et al., 2002; Rottenberg et al., 2007). After watching the film scene, participants rated their mood again. Participants from the Immunisation group and the control group did not watch the film sequence after the first mood rating. Instead, they worked on a distraction task of equal length developed by Nolen-Hoeksema and Morrow (1993), in which participants are to imagine 17 neutral places or objects (e.g., "Imagine looking at the shiny surface of a trumpet", "Think about a boat slowly crossing the Atlantic", "Think about the expression on the face of the Mona Lisa").

\section{Performance Test and Performance Feedback}

After undergoing the mood induction vs. distraction, participants completed the Test of EMotional INTelligence (TEMINT) by Schmidt-Atzer and Bühner (2002), a performance test in which participants are to empathise with other people and guess their emotional states in twelve different situations. To positively disconfirm participants' initial negative expectations, participants received standardised performance feedback suggesting that they were among the best $12-18 \%$ of all participants in this test. This "dose" of feedback was selected based on two aspects that had to be balanced. On the one hand, in order to entail an expectation 
adjustment (in healthy people), the feedback needs to be perceived as positive (i.e. suggesting that the performance was above average). On the other hand, it must not appear suspiciously positive in order to prevent serious doubts concerning its credibility and thereby the cover story of the study. Therefore, we refrained from using overly positive feedback (i.e., best 1\%). Previous work confirmed that this dose of feedback was appropriate to elicit a positive adjustment of initial expectations in non-clinical samples (Kube, Rief, et al., 2019a, b, c; Kube et al., 2018). The TEMINT was selected as a performance test for this paradigm primarily because it is very difficult for participants to evaluate their own performance in this test, which is important for the performance feedback received to appear credible, as validated in previous work (Kube et al., 2018).

\section{Cognitive Immunisation Manipulation}

After completing the test and receiving the last feedback for their performance, participants from the groups Immunisation and Immunisation + Affect received a standardised information text with the aim of triggering the use of cognitive immunisation strategies. In particular, this immunisationpromoting manipulation aimed to elicit an appraisal of the positive feedback typical of people with depression, i.e. to regard good performance as an exception or to question its relevance. Participants were informed that several scientific studies had not found any relation between the performance on the TEMINT and professional success, measurable on both subjective (e.g. work satisfaction) and objective measures (e.g., higher income). Similarly, the manipulation suggested that the results of the TEMINT would not allow any prediction about persons' satisfaction with their private living situation including social relationships. As demonstrated previously in people with depression (Kube, Rief, et al., 2019a, b, c), we hypothesised that this (fake) information about the TEMINT would promote the engagement in cognitive immunisation strategies because the validity and utility of the expectation-disconfirming experience were explicitly questioned.

After completing the test procedure, the second assessment of participants' expectations was performed, followed by several other measures. Finally, participants were debriefed with respect to the true purpose of the study. Figure 1 illustrates the entire study procedure.

\section{Measures}

\section{Performance Expectations}

For the assessment of participants' performance expectations, a previously developed and validated four-item scale was used (Kube, Kirchner, et al., 2019a, b, c; Kube et al.,
2018). This scale distinguishes between task-specific expectations (tied to the particular test procedure; e.g., "I will be successful in working on the tasks from the test") and generalised performance expectations (referring to the performance in unknown situations in general; e.g., "I will be successful in working on unknown tasks in general"), each of which are assessed with two items. Each item is rated on a 7-point Likert scale. Cronbach's alpha was $\alpha=0.84$ for the task-specific expectations subscale and $\alpha=0.86$ for the generalised expectations subscale. As in all previous studies using this measure, changes in generalised expectations were the primary outcome, while task-specific expectations were considered secondary.

\section{Cognitive Immunisation}

To examine differences between the four groups in terms of their engagement in cognitive immunisation strategies, we used the Cognitive Immunisation after Performance Feedback (CIPF) scale. This scale comprises four items to assess the appraisal of a given performance feedback. Two items assess the extent to which participants question the credibility of the expectation-disconfirming feedback (e.g., "The test provides understandable performance feedback" (reversescored)), and two items assess how participants appraise the relevance of the result yielded by the test (e.g., "The competence that was captured by the test is of great importance in my everyday life" (reverse-scored)). High values reflect a greater engagement in cognitive immunisation strategies. The measure has been validated in a previous study and has shown good psychometric properties (Kube, Glombiewski, et al., 2019a, b, c). In the present study, Cronbach's alpha of the CIPF scale was $\alpha=0.72$.

\section{Positive and Negative Affect}

We used the Positive and Negative Affect Scale (PANAS) by Watson, Clark, and Tellegen (1988) Watson et al. (1988), a brief measure of current mood state that has been used to assess positive and negative emotions independently. The PANAS comprises twenty adjectives, ten of which describing positive emotional states (e.g. excited, proud, and inspired) and ten describing negative emotional states (e.g. distressed, jittery, and upset). Participants noted the extent to which they have felt each emotion in the current situation, using a numerical scale ranging from 0 (not at all) to 10 (extremely strong). The PANAS has shown very good psychometric properties in previous studies (Crawford \& Henry, 2004; Terracciano et al., 2003; Watson et al., 1988). In our sample, Cronbach's alpha of the positive affect (PA) subscale was $\alpha=0.87$ before the mood induction and $\alpha=0.91$ thereafter. Cronbach's alpha of the negative affect 


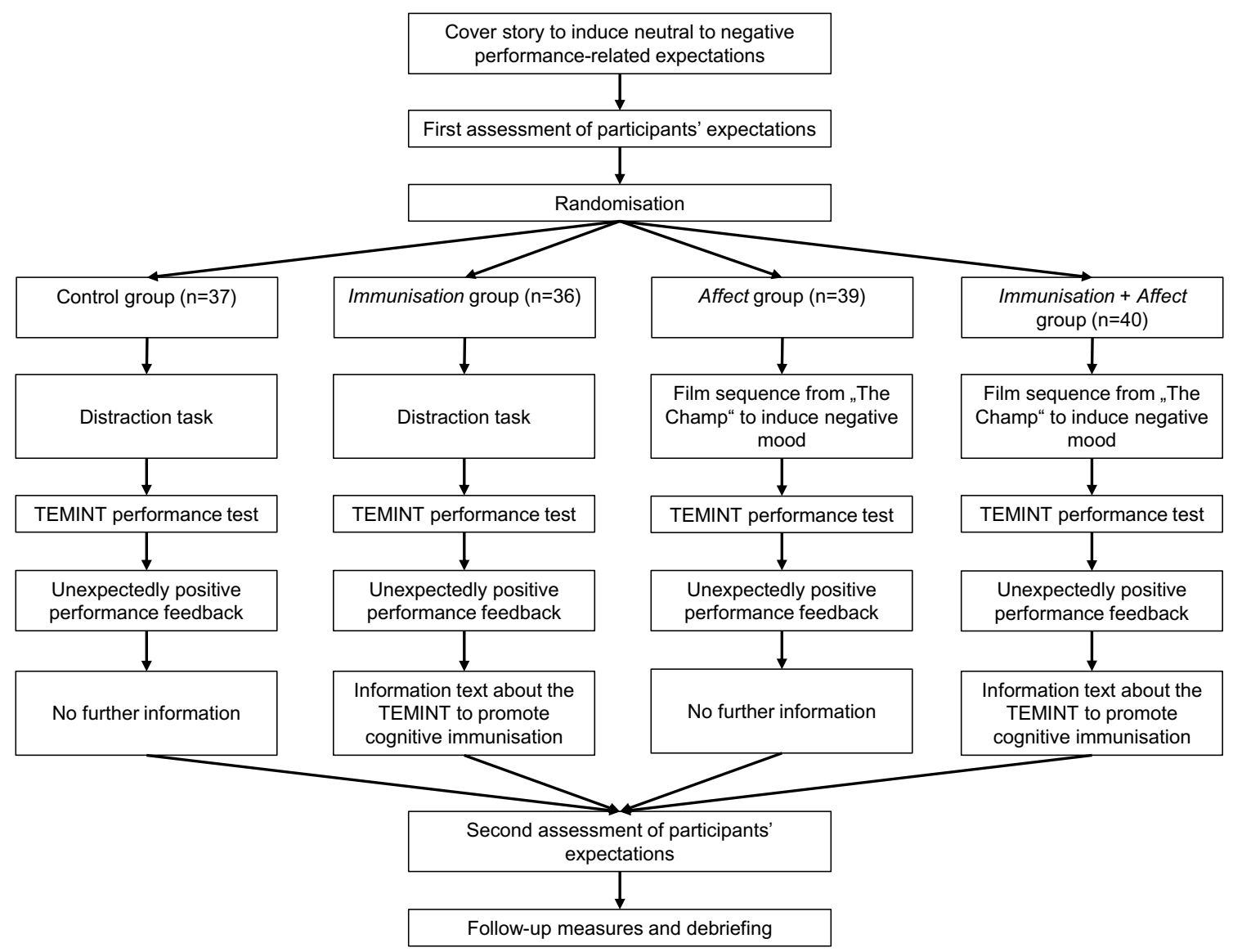

Fig. 1 Design of the present study. After inducing neutral to negative expectations about one's ability to work successfully on an unknown test, participants' expectations were assessed for the first time. Next, participants underwent either a negative mood induction or distraction task and their mood was assessed before and after. Subsequently, participants perform the Test of Emotional Intelligence (TEMINT), on which they receive standardised performance feedback that is

(NA) subscale was $\alpha=0.83$ before the mood induction and $\alpha=0.84$ thereafter.

\section{Depressive Symptoms}

Depressive symptoms were assessed using the second edition of the Beck Depression Inventory (BDI-II; (Beck et al., 1996), comprising 21 items to assess depressive symptoms on a four-point scale ranging from 0 to 3 . The sum score ranges between 0 and 63 with lower values indicating fewer depressive symptoms. In our sample, Cronbach's alpha of the BDI-II was $\alpha=0.88$.

\section{Socio-demographics}

Socio-demographic variables, including age, gender, education, and employment status, were assessed using a brief self-report questionnaire. intended to positively disconfirm their previous expectations. Before assessing participants' expectations again, two groups received a manipulation aimed to promote cognitive immunisation by suggesting that the positive test result would not be valid and relevant for everyday life. After completing some further questionnaires, participants were debriefed

\section{Other Measures}

To examine the potential influence of possible confounding variables, we assessed dispositional optimism and ruminative response style. Dispositional optimism was assessed using the German version of the Life Orientation Test Revised (LOT-R) (Glaesmer et al., 2008). The LOT-R comprises 10 items, of which four items are distractor items. High values of the sum score of the LOT-R reflect a more optimistic view of one's future. In addition, we used the German version of the Response Style Questionnaire (RSQ) (Kühner et al., 2007). The RSQ contains 32 items, each asking participants how they tend to react when they are feeling sad or upset. 21 items assess a ruminative response style (RSQ_RUM), with higher values indicating a more pronounced ruminative response, and 11 items assess the extent to which participants try to distract them from negative mood (RSQ_DIS). 
Table 1 Sample characteristics of study participants

\begin{tabular}{|c|c|c|c|c|}
\hline Variable & Control Group $(n=37)$ & Affect $(n=39)$ & Immunisation $(n=36)$ & $\begin{array}{l}\text { Immunisa- } \\
\text { tion + Affect } \\
(n=40)\end{array}$ \\
\hline Age in years, $M(S D)$ & $22.76(2.63)$ & $24.26(5.27)$ & $23.19(5.87)$ & $22.25(1.79)$ \\
\hline BDI-II sum score, $\mathrm{M}$ (SD) & $12.10(7.09)$ & $10.69(7.91)$ & $10.68(7.04)$ & $12.69(8.71)$ \\
\hline \multicolumn{5}{|l|}{ Sex, N (\%) } \\
\hline Male & $6(16.2)$ & $7(17.9)$ & $6(16.7)$ & $12(30.0)$ \\
\hline Female & $31(83.8)$ & $32(82.1)$ & $30(83.3)$ & $28(70.0)$ \\
\hline \multicolumn{5}{|l|}{ Educational level, N (\%) } \\
\hline Secondary education & 0 & 0 & 0 & $1(2.5)$ \\
\hline High school degree & $32(86.5)$ & $25(64.1)$ & $30(83.3)$ & $32(80)$ \\
\hline University degree & $5(13.5)$ & $14(35.9)$ & $6(17.7)$ & $7(17.5)$ \\
\hline \multicolumn{5}{|l|}{ Employment status } \\
\hline Full-time working & $2(5.4)$ & $4(10.3)$ & $5(13.9)$ & $6(15.0)$ \\
\hline Part-time working & $8(21.6)$ & $2(5.1)$ & $6(16.7)$ & $4(10.0)$ \\
\hline Homemaker & 0 & $1(2.6)$ & 0 & 0 \\
\hline In training & $22(49.5)$ & $30(76.9)$ & $25(69.4)$ & $27(67.5)$ \\
\hline Unemployed & $5(13.5)$ & $2(5.1)$ & 0 & $3(7.5)$ \\
\hline
\end{tabular}

$M$ mean, $S D$ standard deviation, $N$ number, $B D I-I I$ beck depression inventory

All measures were presented in German language and were completed online via the commercial survey platform Unipark®.

\section{Statistical Procedure}

After the pre-analyses (which are in detail reported in the supplemental material), we performed a 2 (Time: before feedback vs. after feedback) $\times 2$ (Immunisation: immunisation-promoting manipulation vs. no manipulation) $\times 2$ (Affect: induction of negative affect vs. distraction) factorial ANOVA with expectations (generalised or task-specific, respectively) for the main analysis. For the moderation hypothesis, we considered the sum score of depressive symptoms as the independent variable and the group assignment variable for the affect manipulation (negative mood induction vs. distraction) as the moderator variable. The dependent variable was the pre to post change in the generalised performance expectations scale. For all analyses, type-1 error levels were set at 5\%. All analyses were conducted using IBM SPSS Statistics Version 25. For the moderation analysis, we used the PROCESS Macro for SPSS for regression analyses with 10,000 bootstrapping samples.

\section{Results}

\section{Sample Characteristics}

Five participants had to be excluded because of serious doubts concerning the cover story. These participants guessed that the study could be about the "effects of feedback on self-perception". Thus, after excluding these participants, subsequent analyses were based on data from 152 participants (with $n=37$ for the control group; $n=39$ for the Affect group; $n=36$ for the Immunisation group; and $n=40$ for the Immunisation + Affect group). The sociodemographic and clinical characteristics of the four experimental groups are presented in Table 1. As can be seen in Table 1, participants reported on average minimal symptoms of depression, $M=11.40 ; S D=7.70$. Forty-nine participants (32.2\%) reported elevated levels of depression (BDI-II sum score $\geq 14$ ). There were no significant baseline differences between the four groups as can be seen in the supplement.

\section{Manipulation Checks}

\section{Mood Induction vs. Distraction}

The Time by Condition two-factorial ANOVA with negative affect as the dependent variable indicated a significant main effect of Time, $F(1,148)=9.434 ; p=0.003 ; \eta_{p}^{2}=0.060$, with overall less negative affect before the mood induction/ distraction $(M=28.71, S D=15.34)$ than after the mood induction/distraction $(M=31.822, S D=16.18)$. The main effect of Condition was not significant, $F(3,148)=2.620$; $p=0.053 ; \eta_{p}^{2}=0.050$. The Time by Condition interaction was significant, $F(3,148)=23.657 ; p<0.001 ; \eta_{p}^{2}=0.324$. Negative affect significantly increased in the groups Affect $(t(38)=-4.273, p<0.001, d=0.684)$ and Affect + Immunisation $(t(39)=-5.443, p<0.001, d=0.860)$, while 
Table 2 Expectation and cognitive immunisation ratings of the four experimental groups

\begin{tabular}{lllll}
\hline Variable & Control group $(n=37)$ & Affect $(n=39)$ & $\begin{array}{l}\text { Immunisation }(n=36) \\
\text { Immunisa- } \\
\text { tion + Affect } \\
(n=40)\end{array}$ \\
\hline $\begin{array}{l}\text { Task-specific expectations, } M(S D) \\
\text { Pre }\end{array}$ & $8.43(2.52)$ & $8.44(2.44)$ & $8.53(2.66)$ & $11.92(1.96)$ \\
Post & $11.54(1.56)$ & $11.67(1.96)$ & & $8.60(2.56)$ \\
Generalised expectations, $M(S D)$ & & & $8.75(2.37)$ & $11.35(2.14)$ \\
Pre & $8.51(2.66)$ & $8.69(2.52)$ & $10.41(2.59)$ & $10.46(2.21)$ \\
Post & $9.91(2.46)$ & $16.51(4.25)$ & $15.81(5.57)$ & $10.15(2.58)$ \\
CIPF scale total score, $M(S D)$ & $14.70(4.65)$ & $9.33(2.53)$ & $8.56(2.83)$ & $7.58)$ \\
CIPF subscale relevance, $M(S D)$ & $8.08(2.79)$ & $7.18(2.66)$ & $7.25(3.39)$ & $7.35(3.56)$ \\
CIPF subscale credibility, $M(S D)$ & $6.62(2.85)$ & & & \\
\hline
\end{tabular}

$M$ mean, $S D$ standard deviation, $C I P F$ cognitive immunisation after performance feedback

it significantly decreased in the Immunisation group $(t(35)=4.318, p<0.001, d=0.720)$ and the control group $(t(36)=3.003, p=0.005, d=0.493)$.

The Time by Condition two-factorial ANOVA with positive affect as the dependent variable indicated a significant main effect of Time, $F(1,148)=99.831 ; p<0.001$; $\eta_{p}^{2}=0.403$, with overall more positive affect before the mood induction/distraction $(M=65.70, S D=14.83)$ than after the mood induction/distraction $(M=56.80$, $S D=17.71)$. The main effect of Condition was not significant, $F(3,148)=1.749 ; p=0.160 ; \eta_{p}^{2}=0.034$. The Time by Condition interaction was significant, $F(3,148)=31.784$; $p<0.001 ; \eta_{p}^{2}=0.392$. Positive affect significantly decreased in the groups Affect $(t(38)=8.509, p<0.001, d=1.364)$ and Affect + Immunisation $(t(39)=9.348, p<0.001, d=1.478)$, but not in the Immunisation group $(t(35)=0.234, p=0.816$, $d=0.039)$ and the control group $(t(36)=0.168, p=0.868$, $d=0.028)$.

\section{Cognitive Immunisation}

The one-way ANOVA indicated that the four experimental groups did not differ in the CIPF sum scores, $F(3$, $148)=0.922, p=0.432, \eta_{p}^{2}=0.018$, nor did they differ on the two subscales relevance, $F(3,148)=1.424, p=0.238$, $\eta_{p}^{2}=0.028$, and credibility, $F(3,148)=0.407, p=0.748$, $\eta_{p}^{2}=0.008$. The descriptive values for the CIPF ratings are presented in Table 2.

\section{Main Analyses}

\section{Changes in Generalised Expectations}

The Time by Immunisation by Affect three-factorial ANOVA with generalised expectations as the dependent variable indicated a significant main effect of Time,
$F(1,148)=73.248 ; p<0.001 ; \eta_{p}^{2}=0.331$, with overall more optimistic expectations after feedback $(M=10.24$, $S D=2.45)$ than before working on the test $(M=8.62$, $S D=2.52)$. The main effect of Immunisation was not significant, $F(1,148)=0.032 ; p=0.859 ; \eta_{p}^{2}<0.001$, nor was the main effect of Affect, $F(1,148)=0.026 ; p=0.873$; $\eta_{p}^{2}<0.001$. The Immunisation by Affect interaction was not significant either, $F(1,148)=0.711 ; p=0.400$; $\eta_{p}^{2}=0.005$. The Time by Immunisation interaction was not significant, $F(1,148)=0.024 ; p=0.877 ; \eta_{p}^{2}<0.001$, nor was the Time by Affect interaction, $F(1,148)=0.182$; $p=0.670 ; \eta_{p}^{2}=0.001$, or the Time by Immunisation by Affect interaction, $F(1,148)=0.288 ; p=0.592$; $\eta_{p}^{2}=0.002$. Descriptive values for generalised (as well as task-specific) expectations are presented in Table 2.

\section{Changes in Task-Specific Expectations}

The Time by Immunisation by Affect three-factorial ANOVA with task-specific expectations as the dependent variable indicated a significant main effect of Time, $F(1$, $148)=155.144 ; p<0.001 ; \eta_{p}^{2}=0.512$, with more optimistic expectations after feedback $(M=11.61, S D=1.92)$ than before working on the test $(M=8.50, S D=2.52)$. As for generalized expectations, all other effects were not significant, that is, the main effect of Immunisation, $F(1,148)=0.089 ; p=0.766 ; \eta^{2}=0.001$, the main effect of Affect, $F(1,148)=0.116 ; p=0.733 ; \eta_{p}^{2}=0.001$, the Immunisation by Affect interaction, $F(1,148)=0.340$; $p=0.560 ; \eta_{p}^{2}=0.002$, the Time by Immunisation interaction, $F(1,148)=0.040 ; p=0.842 ; \eta_{p}{ }_{p}<0.001$, the Time by Affect interaction, $F(1,148)=0.266 ; p=0.607$; $\eta^{2}{ }_{p}=0.002$, and the Time by Immunisation by Affect interaction, $F(1,148)=0.578 ; p=0.448 ; \eta_{p}^{2}=0.004$. 


\section{Association of depressive symptoms and expectation update}

a Participants who underwent the negative mood induction

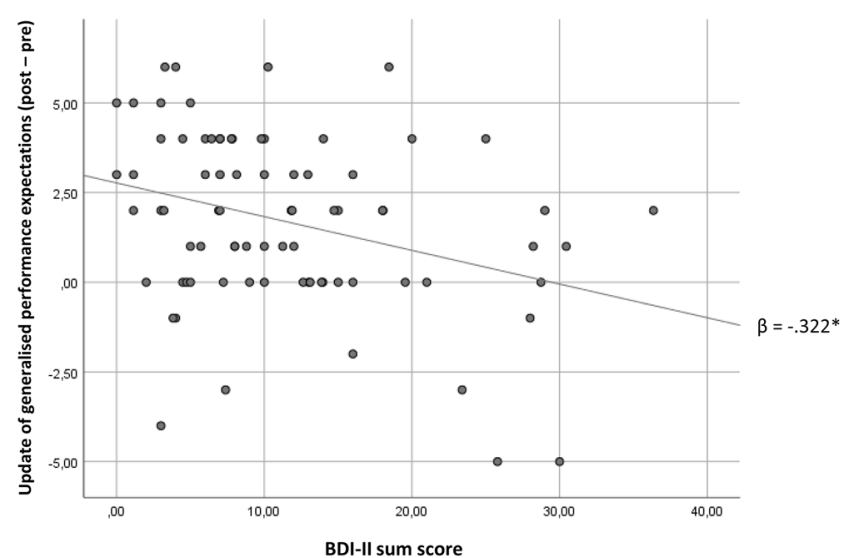

Fig. 2 Results of the moderation analysis examining the relationship between depressive symptoms and expectation update as a function of mood induction vs. distraction. If negative mood was activated (a), depressive symptoms were a significant negative predictor of the

\section{Moderation Analysis}

Results indicate that depressive symptoms were a significant negative predictor of revising generalised expectations, $b=-0.254 ; p<0.001$. As predicted, this effect was moderated by the group assignment in terms of mood induction vs. distraction, $b=-0.160 ; p=0.001$ : depressive symptoms had a particularly negative influence on expectation adjustment if participants underwent the mood induction $(p=0.002)$, whereas they had a non-significant positive influence on expectation adjustment for participants performing the distraction task $(p=0.079)$. This differential influence of depressive symptoms on the revision of expectations, depending on whether or not negative mood was activated, is depicted in Fig. 2.

To follow-up the moderation analysis, we ran an additional analysis in which we added the cognitive immunisation manipulation as a predictor (along with all interaction terms). The results show that cognitive immunisation does not interact with any of the other predictors in the model (i.e., depression, mood, and depression $\times$ mood).

\section{Discussion}

The present study sought to investigate the influence of cognitive immunisation and negative mood on the adjustment of negative performance expectations after receiving unexpectedly positive performance feedback in a non-clinical sample. To this end, we aimed to experimentally induce cognitive immunisation, negative mood, or both, and compared these b Participants who underwent distraction

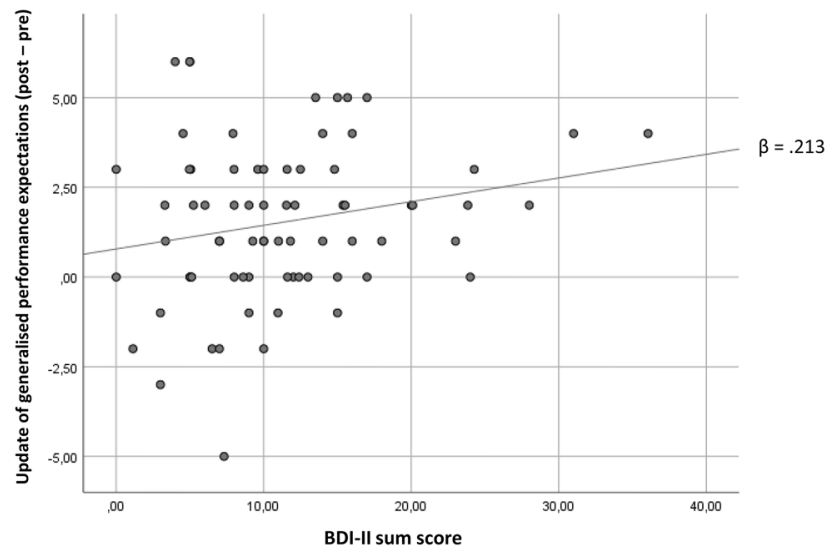

update of generalised performance expectations, whereas depressive symptoms were non-significantly positively associated with expectation update if participants underwent distraction $(\mathbf{b}) . * p<.05$

experimental groups to a control group receiving none of the aforementioned manipulations. The main results show that participants from all groups changed their generalised performance expectations in a positive direction with large effect sizes, regardless of the manipulation received. In particular, neither the main effects of the factors cognitive immunisation and negative affect, nor their interaction was significant. The same pattern of results was found for the secondary outcome, that is, change in task-specific performance expectations. To interpret these results, it is important to consider the results of the manipulation check: on the one hand, as intended, negative affect increased and positive affect decreased in the groups Affect and Immunisation + Affect after watching the sad film sequence; on the other hand, there were no group differences in the sum scores of the immunisation scale, suggesting that it was not possible to manipulate cognitive immunisation through the experimental manipulation used in the groups Immunisation and Immunisation + Affect.

Our first aim was to examine whether cognitive immunisation could also be induced in a non-clinical sample, thereby informing our understanding of whether this cognitive mechanism is specific to depression. Previous studies using clinical samples found that the modulation of cognitive immunisation significantly impacted the revision of negative expectations after unexpectedly positive information; specifically, promoting cognitive immunisation through experimental manipulations prevented depressed people from revising negative expectations after positive feedback (Kube, Rief, et al., 2019a, b, c), while the inhibition of cognitive immunisation facilitated the adjustment of negative 
expectations (Kube, Glombiewski, et al., 2019a, b, c). In the present study, however, the immunisation-promoting manipulation did not lead to an increased engagement in cognitive immunisation strategies, nor did it affect the adjustment of expectations. In other words, the present study extends previous research findings that healthy people modify their expectations in accordance with positive feedback (Kube, Rief, et al., 2019a, b, c; Kube et al., 2018) by demonstrating that this positive adjustment of expectations holds even if the relevance of the positive feedback is explicitly questioned. Thus, in relation to our primary aim, the present findings do not support the hypothesis that cognitive immunisation can be induced in a non-clinical sample as well. To further examine the specificity of cognitive immunisation to depression, it would be valuable for future research to compare people with depression with other clinical groups in terms of their engagement in cognitive immunisation strategies.

These findings add to the literature on cognitive biases, where previous work has shown that although attentional, interpretation, and memory biases are typical of clinically depressed people, such biases could not consistently be observed or experimentally induced in non-clinical samples (Hallion \& Ruscio, 2011; Hertel \& Mathews, 2011). The null effects of the present cognitive immunisation manipulation can also be linked to research into the optimism bias. Specifically, research has demonstrated that belief updating in healthy people is optimistically biased, meaning that they update their beliefs selectively in response to good news while discarding bad news (Sharot, 2011; Sharot, GuitartMasip, et al., 2012; Sharot, Korn, et al., 2011); this bias was found to be absent in clinically depressed people (Garrett et al., 2014). For example, in an experimental study by Korn et al. (2014), healthy people adjusted their expectations about future life events more towards desirable vs. undesirable information, while this optimistic bias was absent in people with depression. Thus, interpreting the present findings in view of this optimism bias, it seems that people without depressive symptoms unswervingly correct negative expectations after receiving novel positive experiences and are not susceptible to devaluing them through cognitive immunisation strategies. In other words, healthy people tend to interpret novel environmental information in a favourable and self-worth stabilising manner, which is also supported by well-established findings from social and personality psychology on self-concept stability (Swann \& Hill, 1982; Swann \& Read, 1981a, 1981b).

However, another possible explanation for the null effects of the cognitive immunisation manipulation might be that it was simply not effective or suitable for this sample. Specifically, the information text provided to the participants stressed e.g. that the TEMINT was not shown to be predictive of professional success (i.e. higher income), and it is conceivable that this information was not relevant to this university student sample as it may have been "too far away" from their perspective. Possibly, this lack of relevance might also explain why the Immunisation by Depression interaction from the supplemental three-way ANOVA was not significant, in contrast to a previous study which used an older sample (Kube, Rief, et al., 2019a, b, c). Finally, a note of methodology: the present experiment was designed to test the hypothesis that cognitive immunisation can be triggered in a non-clinical sample, which was not confirmed by the results; if researchers in future studies were interested in testing the reverse hypothesis that healthy people are "immune" to cognitive immunisation manipulations, this would be the test of a null hypothesis, requiring other statistical procedures.

With respect to the secondary purpose of the present study, that is, the role of negative mood in belief updating, we found that the presentation of a brief film sequence was able to induce negative affect and reduce positive affect ${ }^{3}$; this, however, affected the adjustment of negative performance expectations only among people with elevated symptoms of depression, as indicated in the moderation analysis: overall, depressive symptoms were a negative predictor of expectation adjustment (as also shown in previous work), and this effect was particularly pronounced if participants underwent a negative mood induction before receiving positive feedback. Thus, the results indicate that even in a nonclinical sample, depressive symptoms are associated with impaired processing of unexpectedly positive information if negative affect is amplified by negative mood induction. In people without elevated symptoms of depression, the negative mood induction did not affect the adjustment of their expectations according to the positive feedback received. This differential effect of the negative mood induction could be interpreted in such a way that in people without depressive symptoms, negative mood as induced by the film sequence normally fades away after a few minutes (Gross \& Levenson, 1995; Rottenberg et al., 2002, 2007); in people with increased depressive symptoms, on the other hand, this negative mood tends to persist and thus hinders processing new positive information.

Finally, the present study also allowed an investigation of whether the combined induction of negative mood and cognitive immunisation has particularly detrimental effects on belief updating, beyond the independent effects of each

\footnotetext{
${ }^{3}$ Of note, the results of the manipulation check for negative affect showed not only that the negative mood induction increased negative affect, but also that negative affect decreased in participants who underwent distraction. Although the latter was not the primary purpose of the present study, this finding is consistent with previous work indicating positive effects of distraction on negative affect (Joormann, Siemer, \& Gotlib, 2007; Rusting \& Nolen-Hoeksema, 1998; Van Dillen \& Koole, 2007).
} 
manipulation. The non-significant interaction effect indicates that a combination of a mood and cognitive manipulation does not boost their individual effects. Most likely, this is due to the failure of the cognitive immunisation manipulation to actually induce a negative appraisal of the positive feedback received, as discussed above.

\section{Limitations}

One limitation is that we examined cognitive immunisation and negative mood only in the context of performancerelated expectations. To extend our findings, it would be important to examine the effects of these factors in additional areas beyond performance, e.g., social interactions. Moreover, we focused only on the adjustment of negative expectations after receiving unexpectedly positive information. It would be interesting in future studies to the examine the influence of cognitive immunisation and negative mood on changes in positive expectations after disconfirming negative information. This can also help further examine whether cognitive immunisation is a mechanism specific to depression (or other mental health problems) or whether it can also be found in healthy people. In addition, as highlighted above, a further limitation of the present cognitive immunisation manipulation was that it was tied specifically to the relevance of positive performance feedback; hence, it would be valuable if future studies using similar interventions would aim to target cognitive immunisation in additional contexts. Further limitations refer to the sample: in our study, no pre-selection of people with varying levels of depressive symptoms was conducted; hence, the discussion of the results of the moderation analyses regarding depressive symptoms needs to be interpreted in the context of a sample with continuously varying levels of depressive symptoms. Consequently, future studies may examine the effects of cognitive immunisation and negative mood manipulations by directly comparing a healthy vs. clinical sample, including more sophisticated diagnostic procedures beyond self-report questionnaires. Due to this limitation, we considered depressive symptoms mainly in the moderation analysis allowing to examine the influence of depressive symptoms dimensionally, and only briefly mentioned that similar results could be obtained when considering the presence of elevated depressive symptoms dichotomously.

\section{Conclusions}

The present findings have two important implications: first, people who are in good mental health do not seem to be susceptible to devaluing positive information, but interpret novel environmental information in a favourable and self-worth stabilising way. These findings can be linked to the literature on an optimism bias in healthy people. Second, even in a non-clinical sample, depressive symptoms hinder positive information processing which is particularly pertinent when negative mood is activated.

Acknowledgements We are very grateful to Edith Stadlmeier and Julia Braun, two master students who contributed to the conduction of the study.

Funding Open Access funding enabled and organized by Projekt DEAL. This study was funded by Universität Koblenz-Landau.

\section{Compliance with Ethical Standards}

Conflict of Interest Tobias Kube and Julia Anna Glombiewskil declare that they have no conflicts of interest.

Ethical Approval All procedures performed in studies involving human participants were in accordance with the ethical standards of the institutional and/or national research committee and with the 1964 Helsinki declaration and its later amendments or comparable ethical standards.

Informed Consent Informed consent was obtained from all individual participants included in the study.

Animal Rights No animal studies were carried out by the authors for this article.

Open Access This article is licensed under a Creative Commons Attribution 4.0 International License, which permits use, sharing, adaptation, distribution and reproduction in any medium or format, as long as you give appropriate credit to the original author(s) and the source, provide a link to the Creative Commons licence, and indicate if changes were made. The images or other third party material in this article are included in the article's Creative Commons licence, unless indicated otherwise in a credit line to the material. If material is not included in the article's Creative Commons licence and your intended use is not permitted by statutory regulation or exceeds the permitted use, you will need to obtain permission directly from the copyright holder. To view a copy of this licence, visit http://creativecommons.org/licenses/by/4.0/.

\section{References}

Beck, A. T., Steer, R. A., Ball, R., \& Ranieri, W. F. (1996). Comparison of beck depression inventories-IA and-II in psychiatric outpatients. Journal of Personality Assessment, 67(3), 588-597.

Brand, S., Reimer, T., \& Opwis, K. (2007). How do we learn in a negative mood? Effects of a negative mood on transfer and learning. Learning and Instruction, 17(1), 1-16.

Crawford, J. R., \& Henry, J. D. (2004). The Positive and Negative Affect Schedule (PANAS): Construct validity, measurement properties and normative data in a large non-clinical sample. British Journal of Clinical Psychology, 43(3), 245-265.

Eshel, N., \& Roiser, J. P. (2010). Reward and punishment processing in depression. Biological Psychiatry, 68(2), 118-124.

Everaert, J., Bronstein, M. V., Cannon, T. D., \& Joormann, J. (2018) Looking through tinted glasses: Depression and social anxiety are related to both interpretation biases and inflexible negative interpretations. Clinical Psychological Science, 6(4), 517-528.

Festinger, L. (1962). A theory of cognitive dissonance. Palo Alto, CA: Stanford University Press. 
Forgas, J. P. (2013). Don't worry, be sad! On the cognitive, motivational, and interpersonal benefits of negative mood. Current Directions in Psychological Science, 22(3), 225-232.

Garrett, N., Sharot, T., Faulkner, P., Korn, C. W., Roiser, J. P., \& Dolan, R. J. (2014). Losing the rose tinted glasses: Neural substrates of unbiased belief updating in depression. Frontiers in Human Neuroscience. https://doi.org/10.3389/fnhum .2014.00639.

Glaesmer, H., Hoyer, J., Klotsche, J., \& Herzberg, P. Y. (2008). Die deutsche version des Life-Orientation-Tests (LOT-R) zum dispositionellen optimismus und pessimismus. Zeitschrift für Gesundheitspsychologie, 16(1), 26-31.

Gross, J. J., \& Levenson, R. W. (1995). Emotion elicitation using films. Cognition \& emotion, 9(1), 87-108.

Hallion, L., \& Ruscio, A. (2011). A meta-analysis of the effect of cognitive bias modification on anxiety and depression. Psychological Bulletin, 137(6), 940-958.

Hammer, L. B., \& Stone-Romero, E. F. (1996). Effects of mood state and favorability of feedback on reactions to performance feedback. Perceptual and Motor Skills, 83(3), 923-934.

Hertel, P. T., \& Mathews, A. (2011). Cognitive bias modification: Past perspectives, current findings, and future applications. Perspectives on Psychological Science, 6(6), 521-536.

Joormann, J., Siemer, M., \& Gotlib, I. H. (2007). Mood regulation in depression: Differential effects of distraction and recall of happy memories on sad mood. Journal of Abnormal Psychology, 116(3), 484-490.

Korn, C. W., Sharot, T., Walter, H., Heekeren, H. R., \& Dolan, R. J. (2014). Depression is related to an absence of optimistically biased belief updating about future life events. Psychological Medicine, 44(3), 579-592. https://doi.org/10.1017/s003329171 3001074 .

Kube, T., Glombiewski, J. A., Gall, J., Toussaint, L., Gärtner, T., \& Rief, W. (2019a). How to modify persisting negative expectations in major depression? An experimental study comparing three strategies to inhibit cognitive immunization against novel positive experiences. Journal of Affective Disorders, 250C, 231-240. https://doi.org/10.1016/j.jad.2019.03.027.

Kube, T., Kirchner, L., Rief, W., Gärtner, T., \& Glombiewski, J. A. (2019b). Belief updating in depression is not related to increased sensitivity to unexpectedly negative information. Behaviour Research and Therapy, 123C, 103509. https://doi. org/10.1016/j.brat.2019.103509.

Kube, T., Rief, W., Gollwitzer, M., Gärtner, T., \& Glombiewski, J. A. (2019c). Why dysfunctional expectations in depression persistResults from two experimental studies investigating cognitive immunization. Psychological Medicine, 49(9), 1532-1544. https ://doi.org/10.1017/S0033291718002106.

Kube, T., Rief, W., Gollwitzer, M., \& Glombiewski, J. A. (2018). Introducing an EXperimental Paradigm to investigate Expectation Change (EXPEC). Journal of Behavior Therapy and Experimental Psychiatry, 59, 92-99. https://doi.org/10.1016/j. jbtep.2017.12.002.

Kube, T., Schwarting, R., Rozenkrantz, L., Glombiewski, J. A., \& Rief, W. (2020). Distorted cognitive processes in major depression: A predictive processing perspective. Biological psychiatry, 87(5), 388-398. https://doi.org/10.1016/j.biops ych.2019.07.017.

Kuehner, C., Huffziger, S., \& Liebsch, K. (2009). Rumination, distraction and mindful self-focus: Effects on mood, dysfunctional attitudes and cortisol stress response. Psychological Medicine, $39(2), 219-228$.

Kühner, C., Huffziger, S., \& Nolen-Hoeksema, S. (2007). Response styles questionnaire: RSQ-D; deutsche version. Göttingen: Hogrefe.
Liknaitzky, P., Smillie, L. D., \& Allen, N. B. (2017). Out-of-the-blue: Depressive symptoms are associated with deficits in processing inferential expectancy-violations using a novel cognitive rigidity task. Cognitive Therapy and Research, 41(5), 757-776. https ://doi.org/10.1007/s10608-017-9853-x.

Lyubomirsky, S., Caldwell, N., \& Nolen-Hoeksema, S. (1998). Effects of ruminative and distracting responses to depressed mood on retrieval of autobiographical memories. Journal of Personality and Social Psychology, 75(1), 166-177.

Mathews, A., \& Mackintosh, B. (2000). Induced emotional interpretation bias and anxiety. Journal of Abnormal Psychology, 109(4), 602-615.

Nolen-Hoeksema, S., \& Morrow, J. (1993). Effects of rumination and distraction on naturally occurring depressed mood. Cognition \& Emotion, 7(6), 561-570.

Nolen-Hoeksema, S., Morrow, J., \& Fredrickson, B. L. (1993). Response styles and the duration of episodes of depressed mood. Journal of Abnormal Psychology, 102(1), 20-28.

Nolen-Hoeksema, S., Parker, L. E., \& Larson, J. (1994). Ruminative coping with depressed mood following loss. Journal of Personality and Social Psychology, 67(1), 92-104.

Rief, W., Glombiewski, J. A., Gollwitzer, M., Schubo, A., Schwarting, R., \& Thorwart, A. (2015). Expectancies as core features of mental disorders. Current Opinion in Psychiatry, 28(5), 378-385. https://doi.org/10.1097/yco.0000000000000184.

Rief, W., \& Joormann, J. (2019). Revisiting the cognitive model of depression: The role of expectations. Clinical Psychology in Europe, 1(1), 1-11.

Rottenberg, J., Kasch, K. L., Gross, J. J., \& Gotlib, I. H. (2002). Sadness and amusement reactivity differentially predict concurrent and prospective functioning in major depressive disorder. Emotion, 2(2), 135-146.

Rottenberg, J., Ray, R. D., \& Gross, J. J. (2007). Emotion elicitation using films. In J. A. Coan \& J. J. B. Allen (Eds.), Handbook of emotion elicitation and assessment (pp. 9-28). New York: Oxford University Press.

Rusting, C., \& Nolen-Hoeksema, S. (1998). Regulating responses to anger: effects of rumination and distraction on angry mood. Journal of Personality and Social Psychology, 74(3), 790-803.

Schmidt-Atzer, L., \& Bühner, M. (2002). Development of a performance measure of emotional intelligence. Berlin, Germany: Paper presented at the Forty-third Congress of the German Psychological Society.

Sharot, T. (2011). The optimism bias. Current Biology, 21(23), R941-R945.

Sharot, T., \& Garrett, N. (2016). Forming beliefs: Why valence matters. Trends in Cognitive Sciences, 20(1), 25-33.

Sharot, T., Guitart-Masip, M., Korn, C. W., Chowdhury, R., \& Dolan, R. J. (2012). How dopamine enhances an optimism bias in humans. Current Biology, 22(16), 1477-1481.

Sharot, T., Korn, C. W., \& Dolan, R. J. (2011). How unrealistic optimism is maintained in the face of reality. Nature Neuroscience, 14(11), 1475-1482.

Stange, J. P., Alloy, L. B., \& Fresco, D. M. (2017). Inflexibility as a vulnerability to depression: A systematic qualitative review. Clinical Psychology: Science and Practice, 24(3), 245-276.

Swann, W. B., \& Hill, C. A. (1982). When our identities are mistaken: Reaffirming self-conceptions through social interaction. Journal of Personality and Social Psychology, 43(1), 59-66.

Swann, W. B., \& Read, S. J. (1981a). Acquiring self-knowledge: The search for feedback that fits. Journal of Personality and Social Psychology, 41(6), 1119-1128.

Swann, W. B., \& Read, S. J. (1981b). Self-verification processes: How we sustain our self-conceptions. Journal of Experimental Social Psychology, 17(4), 351-372. 
Terracciano, A., McCrae, R. R., \& Costa, P. T. (2003). Factorial and construct validity of the Italian Positive and Negative Affect Schedule (PANAS). European Journal of Psychological Assessment, 19(2), 131-141.

Van Dillen, L. F., \& Koole, S. L. (2007). Clearing the mind: A working memory model of distraction from negative mood. Emotion, 7(4), 715-723.

Watson, D., Clark, L. A., \& Tellegen, A. (1988). Development and validation of brief measures of positive and negative affect: The PANAS scales. Journal of Personality and Social Psychology, 54(6), 1063-1070.

Yoon, K. L., \& Joormann, J. (2012). Is timing everything? Sequential effects of rumination and distraction on interpersonal problem solving. Cognitive Therapy and Research, 36(3), 165-172.
Ziegler, R. (2010). Mood, source characteristics, and message processing: A mood-congruent expectancies approach. Journal of Experimental Social Psychology, 46(5), 743-752.

Ziegler, R. (2013). Mood and processing of proattitudinal and counterattitudinal messages. Personality and Social Psychology Bulletin, 39(4), 482-495.

Publisher's Note Springer Nature remains neutral with regard to jurisdictional claims in published maps and institutional affiliations. 